\title{
Video Article \\ Closed System Cell Culture Protocol Using HYPERStack Vessels with Gas Permeable Material Technology
}

\author{
Kim Titus ${ }^{{ }^{1}}$, Vitaly Klimovich ${ }^{2}$, Mark Rothenberg ${ }^{2}$, Pilar Pardo $^{\star^{2}}$, Allison Tanner ${ }^{{ }^{3}}$, Greg Martin ${ }^{3}$ \\ ${ }^{1}$ Business Development, Corning Life Science \\ ${ }^{2}$ Applications, Corning Life Science \\ ${ }^{3}$ Product Development, Corning Life Science \\ *These authors contributed equally
}

Correspondence to: Kim Titus at titusk@corning.com

URL: https://www.jove.com/video/2499

DOI: doi:10.3791/2499

Keywords: Cellular Biology, Issue 45, cell culture, bioprocess, adherent, primary cell, HYPERStack, closed system, gas permeable, cell therapy, vaccine, scale up

\section{Date Published: $11 / 29 / 2010$}

Citation: Titus, K., Klimovich, V., Rothenberg, M., Pardo, P., Tanner, A., Martin, G. Closed System Cell Culture Protocol Using HYPERStack Vessels with Gas Permeable Material Technology. J. Vis. Exp. (45), e2499, doi:10.3791/2499 (2010).

\section{Abstract}

Large volume adherent cell culture is currently standardized on stacked plate cell growth products when microcarrier beads are not an optimal choice. HYPERStack vessels allow closed system scale up from the current stacked plate products and delivers $>2.5 \mathrm{X}$ more cells in the same volumetric footprint. The HYPERStack vessels function via gas permeable material which allows gas exchange to occur, therefore eliminating the need for internal headspace within a vessel. The elimination of headspace allows the compartment where cell growth occurs to be minimized to reduce space, allowing more layers of cell growth surface area within the same volumetric footprint.

For many applications such as cell therapy or vaccine production, a closed system is required for cell growth and harvesting. The HYPERStack vessel allows cell and reagent addition and removal via tubing from media bags or other methods.

This protocol will explain the technology behind the gas permeable material used in the HYPERStack vessels, gas diffusion results to meet the metabolic needs of cells, closed system cell growth protocols, and various harvesting methods.

\section{Video Link}

The video component of this article can be found at https://www.jove.com/video/2499/

Protocol

\section{HYPERStack Vessel - Gas Permeable Material Technology Background}

1. The HYPERStack Vessel is a multilayered vessel for closed system culturing of cells that relies on gas exchange through a $76.2 \mathrm{micron}$ gas permeable polymer film for cellular metabolism.

2. The HYPERStack vessel differs from more traditional cell culture vessels in that it does not have a "headspace" above the cells inside the vessel. Rather than containing this "headspace" for gas exchange within the vessel, the gas permeable products have air spaces (referred to as "tracheal" spaces) beneath each culture chamber which is open to the atmosphere.

3. This tracheal space enables the cells growing in each cell culture compartment of the vessel to have equivalent gas exchange.

4. The gas permeable film allows gas exchange to occur while maintaining a sterile environment. All fluid manipulations for each of the multiple layers occur through a single entry port.

\section{HYPERStack Vessel Component Summary}

1. The Stackette is the individual cell culture compartment that is made up of the top plate and gas permeable film. The cells are cultured within this compartment.

2. The Liquid Manifold connects each of the 12 stackette layers together within a HYPERStack module. Modules are connected with tubing to form vessels in multiples of 12 layers. The manifold allows the user to make one fluid manipulation to the entire vessel.

3. The Air Manifold also connects the stackette layers together, but it used to displace air from the vessel when fluid additions occur. It contains a fill line for use during filling.

4. The Tracheal Space is the open air space between each stackette layer, allowing gas exchange to occur through each layers' gas permeable film. 
5. The Liquid handling tubing is connected to the liquid manifold and is used to make all closed system fluid manipulations. This component can be customized.

6. The Vent tubing is connected to the air manifold, contains an air filter, and is used to release excess air while maintaining sterility.

7. The chase tubing has a filter and clamp and are connected to the liquid handling tubing and are used to evacuate the fluid from the liquid handling tubing after filling the vessel.

8. The Corning Stack Manipulator or CSM is a handling device to assist in placing the vessels in the correct positions during use.

9. The Filling Wedge is a stainless steel assist used when manually filling the HYPERStack vessels.

\section{Gas Diffusion Results Through Gas Permeable Film Used in the HYPERStack Vessels}

1. In traditional cell growth systems with vented headspace, the oxygen in the media is depleted an average of $50 \%$ over 3 days in culture. (Figure 1)

2. In the same cell growth system, the oxygen gradient in a $3 \mathrm{~mm}$ height of media is almost $50 \%$ greater at the media to headspace junction than it is at the cell layer. (Figure 2)

3. The diffusion of oxygen through the 76.2 micron gas permeable polystyrene film is equal to the diffusion through $2.6 \mathrm{~mm}$ of media. The gas permeable film used in the HYPERStack vessels allows the gas exchange to occur at the cell layer.

4. The $\%$ of oxygen in the tracheal space of the HYPERStack vessels remains constant as cells grow to confluence. (Figure 3 )

\section{Media Bag Preparation}

1. The HYPERStack-12 layer vessel takes $1.3 \mathrm{~L}$ of media and the HYPERStack-36 layer vessel takes 3.9L of media.

2. Prior to inoculating your bagged media, if serum is to be used, tube weld the serum bag to the media bag and mix thoroughly.

3. Clamp off approximately $300 \mathrm{~mL}$ of media in the bag using large bag clamps. This will ensure all inoculated cells are used during filling and none are remaining in the media bag. Place media bag on bag stand.

\section{Inoculating Media}

1. Fill a syringe with tubing attached with cell suspension. Tube weld $3 / 16$ " tubing from a syringe containing cell suspension to the media bag.

2. Inject the Cell Suspension into the Media Bag and Mix well.

\section{Fill Procedure}

1. Tube weld the inoculated media bag to the HYPERStack vessel's $3 / 8$ " liquid handling tubing. (Using Sterile Connects or Multipurpose connects (MPCs), you can also attach the media bag to the vessel).

2. Close the clamps on the liquid handling and chase tubes on the HYPERStack.

3. Place the HYPERStack 36 Layer Vessel into the CSM in the load position; hook the vent filter tube into the holding clamp on the CSM. Tighten the lid and move the CSM to the fill position. By putting the vessel into the correct fill position; the air filter is now in the highest position to prevent wetting during fill operation. The $10^{\circ}$ angle permits equilibration of the liquid to the layers during filling. The HYPERStack-12 layer vessel placed on its side on the filling wedge is in the correct position for filling.

4. Clear air from the fill line by positioning the bag at the same level as the HYPERStack vessel (not higher). Keeping the chase tube clamp closed; open the liquid handling tube clamp and the media bag clamp to allow fluid to enter the vessel.

5. Using the bag stand, raise the media bag to help the cell suspension flow into the vessel.

6. Fill the vessel until the all of the inoculated media enters the vessel; the $300 \mathrm{~mL}$ of media should still remain in the top portion of the media bag. Remove the clamp from the media bag to continue filling the vessel. As liquid approaches the upper air manifold, slow down the fill rate by lowering the media bag in order to prevent over filling. Slowly bring the liquid level to the fill line and clamp the liquid handling tubing.

\section{Isolation Procedure to Lock Liquid Into the Layers}

1. Bring the HYPERStack vessel in the CSM to the isolation position so both sets of manifolds are in the highest position and lower the media bag below the height of the vessel. The HYPERStack-12 layer vessel can be lifted into the isolation position by placing a hand on the side of the vessel and using the other to lift the filling wedge.

2. With the chase filter held in an upright position, open the chase tube clamp. This will empty or chase the media in the liquid handling tube back into the media bag. Once the tubing is empty, close the clamp on the media bag tubing.

3. Keeping the chase tube filter in an upright position, open the clamp on the liquid handling tube on the HYPERStack to allow any remaining liquid to enter and equilibrate in the vessel. Wait 1-2 minutes for this to occur.

4. Turn the HYPERStack vessel in the CSM so that the manifolds are on the left. Lower the vessel to the load position on the CSM. Rocking the HYPEStack-12 layer vessel slightly to the left will permit the removal of the filling wedge. Close the clamps on both the chase and liquid handling tubing.

5. The media bag may now be removed from the vessel or it may remain attached for use later in the harvest procedure. To store the attached media bag, roll the empty bag and any remaining media in the bag, and place under the retaining bands in the storage tray of the HYPERStack vessel.

6. Move the HYPERStack vessel to the incubator. When carrying the vessel care should be taken to keep liquid from entering the air vent filter This is accomplished by tipping the manifold end of the HYPERStack slightly upward. 


\section{Harvest Protocol}

1. To begin the harvest procedure, tube weld the cell dissociation solution and quench bags together to form the harvesting bag assembly. Ensure all tubing clamps are closed. (Use cartoon to demonstrate)

2. Remove HYPERStack vessel from the incubator, release the media bag from under the retaining bands, and hang on the bag stand. Place the vessel in the CSM in the load position and tighten the lid to secure the vessel. Hook the vent tubing into the holding clamp and move the CSM to the fill position.

3. Making sure the media bag on the bag stand is hanging lower than the HYPERStack; open the clamp on the media bag tubing and the vessel's liquid handling tubing to allow media to flow into the attached bag.

4. Once the vessel is about $3 / 4$ empty, change the CSM settings to the final empty position. When the HYPERStack vessel and liquid handling tubing are empty, lift the tubing to chase the media past the clamp on the spent media bag tubing, then close both the clamp on the liquid handling tubing and the clamp on the spent media bag tubing.

5. Replace the spent media bag with the harvesting bag assembly by tube welding and raise the height of the cell dissociation bag assembly above the height of the HYPERStack using the bag stand.

6. Place the CSM in the equilibration position. Open the cell dissociation solution tube clamp and transfer the solution into the HYPERStack. When the transfer is complete, close the clamps on the liquid handling tubing and the cell dissociation solution tubing.

7. Bring CSM to the load position. By holding the horizontal positioning lever open, gently rock the vessel side to side using the wheel, distributing the solution over the cell layers. The vessel may occasionally be returned to the equilibration position during rocking to maintain equal distribution of the solution. It may not be visually apparent that the layers are completely coated, but the dissociation solution will be equally distributed across the surface of the cells. Once the solution has been adequately distributed, release the lever on the CSM to lock the HYPERStack in the load position. Leave the HYPERStack in this position during the required dissociation time for your cells.

8. Once the cells have detached; using turbidity as a guide, move the CSM to the equilibration position. Care should be taken not to over digest the cells. Open the quench bag clamp and the HYPERStack liquid handling tubing clamp to allow the quench media to enter the vessel. Close the clamp on the HYPERStack liquid handling tubing once the transfer is complete.

9. Return the CSM to the load position and hold the horizontal positioning lever to rock the vessel side to side.

10. Using the bag stand, lower the position of the harvest bag assembly below the level of the HYPERStack. Adjust the CSM to the empty position, open the vessel liquid handling tube clamp and transfer the cell solution back into the quench bag. Return the CSM to the load position and disconnect the harvest bag assembly from the vessel using a tube sealer. The cell solution is ready for processing.

\section{Representative Results}

- Harvesting time needs to be optimized when converting from a standard polystyrene surface to the gas permeable polystyrene surface. Cells tend to release faster on the HYPERStack product.

- To view cells under a microscope the vent and liquid handling tubing needs to be clamped off. Place the HYPERStack vessel upside down, with the manifolds pointing down, on the microscope stage.

- Prevention steps can be taken to avoid wetting the filters during use which will cause them to stop functioning. By not over filling the HYPERStack vessel and keeping the vent and chase tubing elevated when the clamps are open, no wetting should occur.

- The pressure of the closed clamps on the tubing causes it to remain closed after the clamps are opened. Pinching the tubing in the opposite direction will open it.

- Media flow time entering and exiting the HYPERStack vessel via gravity feed is based on the media height difference between the top level of media in the HYPERStack vessel and the top level of media in the media container. The greater distance, the faster the flow. The maximum flow is limited by the rating of the filters to $1.5 \mathrm{~L} / \mathrm{min}$.

- Maintaining proper fill volume in the HYPERStack vessel will prevent air bubble formation in the cell growth chambers. Air bubbles can occur due to sampling or incubating in an unhumidified environment due to evaporative water loss. The sampling amount and frequency as well as incubation conditions will determine if additional fluid should be added by the end user.

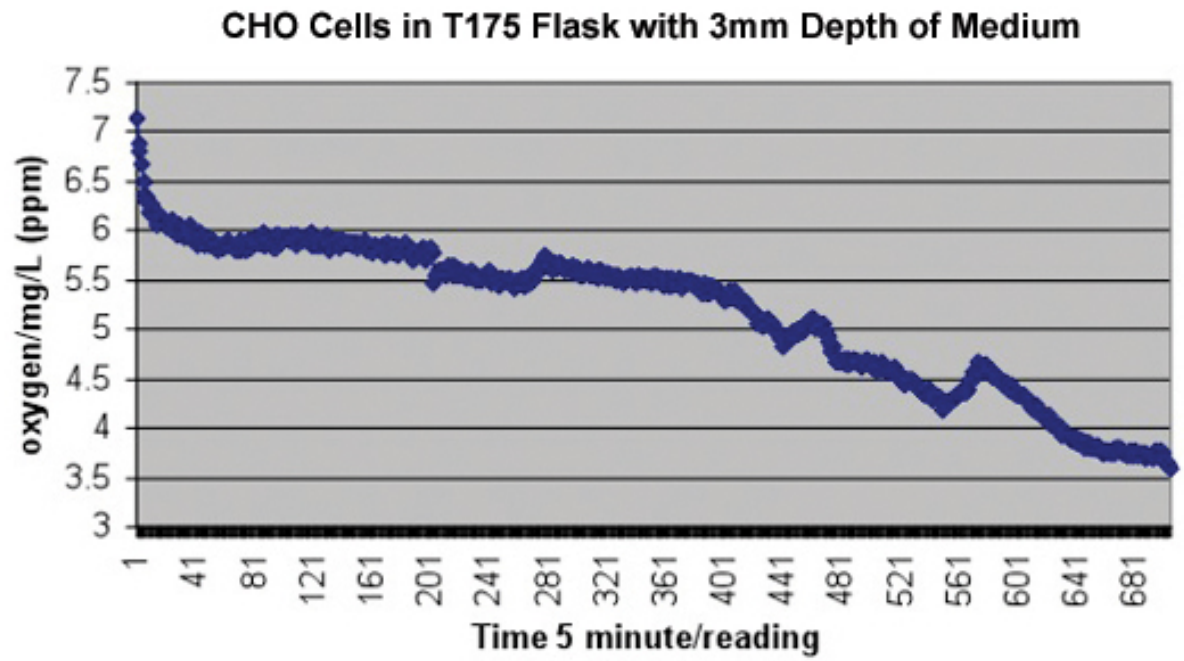


Figure 1. Oxygen depletion of media in a standard cell culture vessel. The figure shows the decrease in $\mathrm{mg} / \mathrm{L}$ of oxygen at the level of the cells over 3 days.

\section{Oxygen Gradient in T-175 Flask with Vent Cap}

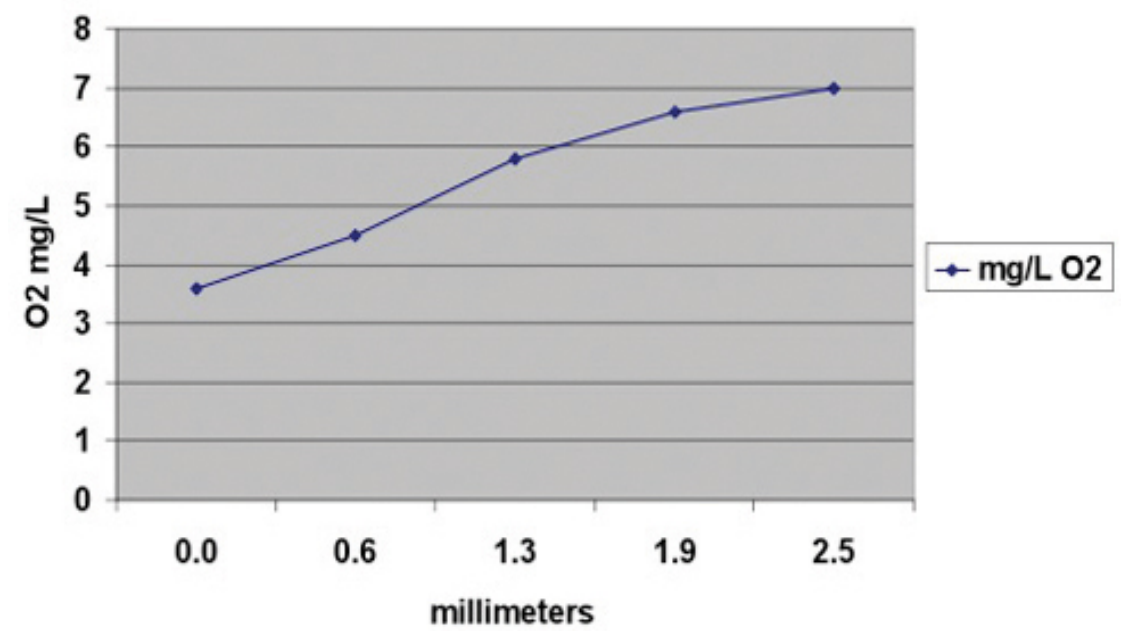

Figure 2. Oxygen gradient in media in a standard cell culture vessel. The figure shows the $\mathrm{mg} / \mathrm{L}$ of Oxygen is greatest at the media to headspace intersection than at the cell layer.

Tracheal Space \% Oxygen: no incubator fan

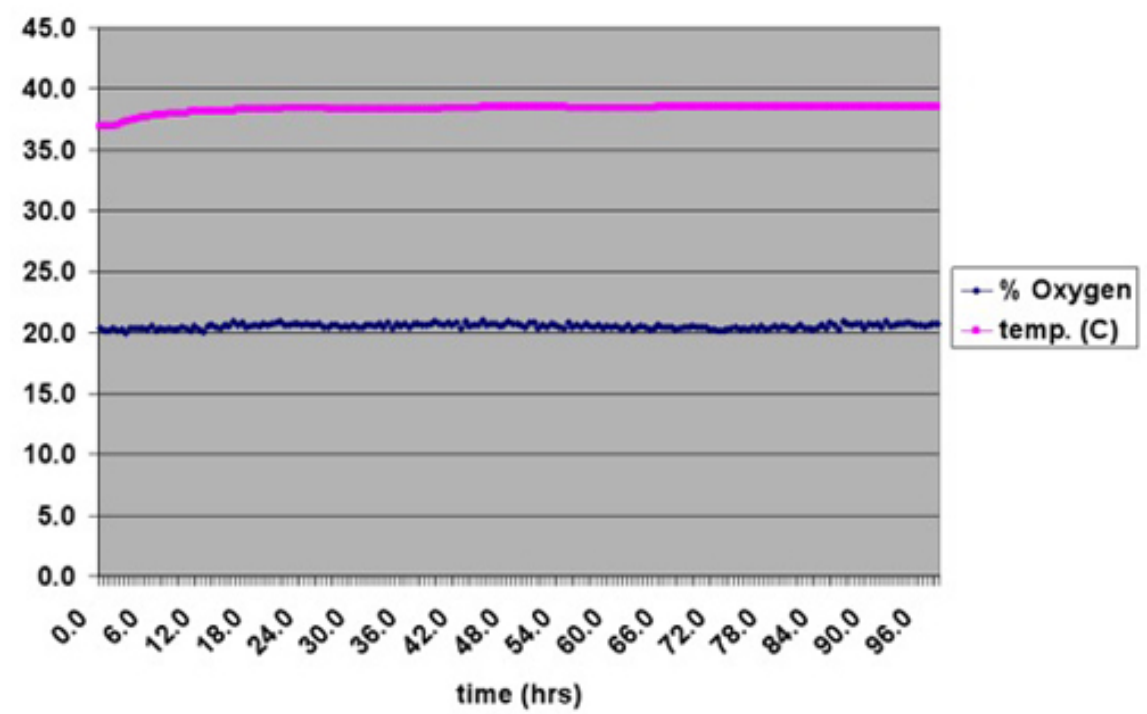

Figure 3. Percentage of Oxygen in the tracheal space of the HYPERStack during cell growth. The figure shows that over 96 hours, the percentage of oxygen between the layers, which supplies each gas permeable film bottom, remains constant. This demonstrates the ability of the cells to access oxygen during growth to confluency.

\section{Discussion}

HYPERStack vessel has successfully grown engineered, primary and stem cells. Fluid additions into the vessel have been conducted via aseptic bag connections by gravity feed and also by pumping from a sterile media container. The vessel provides a greater than $2.5 \mathrm{X}$ increase in growth area vs. a stacked plate product of the same volumetric footprint. The HYPERStack-12 has $6,000 \mathrm{~cm}^{2}$ growth area, the HYPERStack- 36 has $18,000 \mathrm{~cm}^{2}$ growth area, and the HYPERStack-120 has $60,000 \mathrm{~cm}^{2}$ of growth area. By increasing the surface area, users are able to produce more cells from the same cell population or lot and reduce variability. They are also able to save space and/or labor by using fewer vessels to achieve the same cell output, or increase cell output without adding people, cleanroom suites, or incubators. The system is produced with low particulate assembly methods and without the use of adhesives. It is a closed system which is preassembled with tubing sets and filters, triple bagged and provided sterile. 


\section{Disclosures}

The HYPERStack vessel gas permeable technology is covered under US Patent \#: 7745209. The authors are employees of Corning Life Sciences, Inc that produces the instrument used in this article.

\section{Acknowledgements}

The authors would like to thank Lonza Walkersville for their assistance with closed system knowledge, bag design, loan of bag sealers and welders, as well as creating the bag to HYPERStack schematics. 